\title{
Herramienta para la generación de mapas de rendimiento en cítricos usando los datos de una plataforma de asistencia a la recolección con sistema de clasificación
}

\author{
M.G. González-González ${ }^{1}$, J. Gómez ${ }^{2}$, V. Alegre ${ }^{1}$, S. López ${ }^{1}$, J. Blasco ${ }^{1}$, S. Cubero ${ }^{1}$, E. Soria ${ }^{2}$, \\ P. Chueca ${ }^{1}$ \\ 1 Instituto Valenciano de Investigaciones Agrarias (IVIA), Valencia, Spain, \\ 2 Intelligent Data Analysis Laboratory (IDAL), Universitat de València, Valencia, Spain.
}

\begin{abstract}
Resumen: El uso de las nuevas tecnologías en la agricultura moderna permite obtener información esencial para la gestión óptima de la explotación del cultivo. Una de sus principales ventajas es la posibilidad de asociar los datos con ubicaciones muy específicas del cultivo. La representación espacial de esta información permite llevar a cabo operaciones agrícolas de manera precisa, consiguiendo así una mayor eficiencia en la gestión del cultivo. Este trabajo se engloba dentro del objetivo de desarrollar una aplicación web de concepto abierto utilizando el lenguaje de programación R, capaz de generar mapas agrícolas que muestren la información capturada del cultivo utilizando diferentes sensores. Una función clave de esta aplicación es la creación interna de una base de datos que enlaza todas las propiedades medidas por cualquier sensor con la información espacial proporcionada por un sistema de posicionamiento global (GPS) sin la necesidad de integrar todos estos componentes en un conjunto. En este trabajo se presenta un caso práctico de la aplicación web utilizando los datos recopilados por un prototipo de plataforma de asistencia a la recolección de cítricos.
\end{abstract}

Palabras clave: $S I G$, software $R$, variabilidad espacial, agricultura de precisión.

\section{Introducción}

El uso de las tecnologías de la información (TIC) en la agricultura de precisión permite optimizar el rendimiento de las explotaciones agrarias. La asociación de la información obtenida en campo por medio de sensores, satélites, sistemas de posicionamiento global (GPS) junto con sistemas de información geográfica $(S I G)$ posibilita la monitorización del rendimiento. Una de las principales tecnologías de agricultura de precisión es la generación de mapas de rendimiento [1]. Son una herramienta clave debido a su utilidad tanto en el desarrollo como en la evaluación de estrategias de gestión de precisión [2]. La representación espacial y temporal de estos datos a través de mapas es una de las técnicas más efectivas para presentar la información de manera útil y comprensible a los agricultores. La combinación de los mapas de rendimiento espaciales generados en diferentes franjas temporales presenta un valor añadido para la toma de decisiones de gestión más estratégica a medio y largo plazo [3].

Para la generación de los mapas con la información obtenida en campo asociada a la información del GPS se recurre a los SIG. Estos sistemas son herramientas informáticas específicas que mediante información espacialmente referenciada posibilitan el almacenamiento, manipulación, edición, análisis y presentación de gran cantidad de datos en diversas formas 


\section{CONGRESO IBÉRICO DE AGROINGENIERÍA \\ X CONGRESSO IBÉRICO DE AGROENGENHARIA \\ 3 - 6 septiembre 2019, Huesca - España}

(gráficos, figuras, mapas, etc.) [4]. En la actualidad existen multitud de programas comerciales SIG y también de gestión agrícola con módulos SIG incorporados (QGIS, AFS, ArcGIS, Trimble $A g$, entre otros). Estos programas suelen ser utilizados por las empresas que prestan servicios a los productores agrícolas en el área de la agricultura de precisión [5]. Algunos investigadores han desarrollado herramientas propias con módulos $\operatorname{SIG}[6,7,8,9,10]$.

Todos estos sistemas necesitan una base de datos espacial para georreferenciar la información en los mapas. Esta base de datos es creada durante la obtención de las propiedades del cultivo mediante un sensor o un conjunto de sensores. Para componer la base de datos internamente, las propiedades del cultivo están vinculadas a las coordenadas espaciales obtenidas de un GPS integrado. Uno de los principales inconvenientes de este tipo de sistemas cerrados es que las mediciones capturadas por otros sensores que no forman parte del conjunto son difíciles de integrar, $\mathrm{y}$, por ende, de ser utilizadas para generar mapas agrícolas combinados.

El presente trabajo se engloba en el marco de un objetivo general en el que se pretende desarrollar una nueva herramienta para monitorizar y generar mapas de rendimiento con información del cultivo obtenida desde diferentes sensores independientes. Esto representará una mejora significativa sobre los anteriores métodos de generación de mapas puesto que enlazará todas las propiedades medidas por cualquier sensor con la información espacial proporcionada por un GPS sin la necesidad de integrar todos estos componentes en un conjunto creando así una base de datos espacial combinada.

En este trabajo se presenta un caso práctico de la aplicación web haciendo uso de datos obtenidos en un ensayo de recolección de cítricos con un prototipo de plataforma móvil de asistencia a la recolección. La aplicación desarrollada tiene la capacidad de procesar, analizar y visualizar la información en un entorno intuitivo y fácilmente manejable. Proporciona información precisa, analiza los datos y crea mapas de rendimiento lo que aumentará la eficiencia de gestión en campo y la trazabilidad de la fruta.

\section{Materiales y métodos}

La aplicación web ha sido desarrollada utilizando el software de código abierto $R$ por su alto potencial para el procesamiento, la extracción de datos y el análisis computacional de grandes cantidades de información.

\subsection{Creación de la base de datos combinada}

Para la creación de la base de datos espacial combinada cada sensor debe generar un archivo que asocie las mediciones capturadas con el momento en que se capturan. En la aplicación se ha de insertar el fichero que contiene los datos de ubicación GPS y los ficheros creados por los diferentes sensores que contienen información sobre el cultivo. Mediante tratamiento y procesado de datos, esta información se vincula en función del tiempo, descartándose los errores en la toma de datos por parte del GPS. La aplicación presenta la base de datos combinada embebida en su sistema.

Las variables contenidas en la base de datos se visualizan agrupadas según: tiempo (fecha y hora), ubicación (longitud, latitud y altitud), informativas (relacionadas con el método de obtención de los datos) y variables de calidad (información sobre el cultivo). Las variables cuantitativas se han medido en el Sistema Internacional de Unidades (SI). Las variables de longitud y latitud se muestran en el World Geodetic System 1984 (WGS84).

Esta base de datos puede ser filtrada mediante los controles presentes en la aplicación. La base de datos espacial, original y filtrada, puede ser descargada en un archivo con formato csv.

\subsection{Georreferenciación de la información y análisis de datos en la aplicación}

Una vez creada la base de datos, la aplicación permite mostrar la información del cultivo en mapas orográficos a nivel de planta, calle (o fila) de cultivo y parcela. Se ha optado por mostrar 


\section{CONGRESO IBÉRICO DE AGROINGENIERÍA \\ X CONGRESSO IBÉRICO DE AGROENGENHARIA \\ 3 - 6 septiembre 2019, Huesca - España}

cada dato georreferenciado sobre un mapa orográfico en función de las variables seleccionadas en lugar de agrupar conjuntos de datos con información de todas las variables en una cuadrícula de resolución determinada [11] ya que, con ello, se pierde la singularidad de los datos o en lugar de la superposición de un mapa de superficie de la distribución del rendimiento [12] con lo que se difuminan los datos perdiendo así información de posibles datos anómalos. Como complemento a los mapas, se muestran las tablas que resumen la información cuantitativa del cultivo según los diferentes niveles seleccionados.

Además, la distribución espacial de la información de acuerdo con las diferentes variables puede analizarse estadísticamente en los diferentes niveles, obteniendo así la variabilidad espacial de las propiedades medidas en el cultivo para ayudar a la toma de decisiones sobre el manejo del cultivo. La información se muestra en diversos gráficos interactivos a modo de apoyo visual, facilitando así la comprensión de la información.

\subsection{Ensayo de recolección para obtención de datos y prueba de la aplicación}

La aplicación ha sido probada utilizando los datos recopilados por un prototipo de plataforma móvil de asistencia a la recolección de cítricos creado en el IVIA (figura 1).

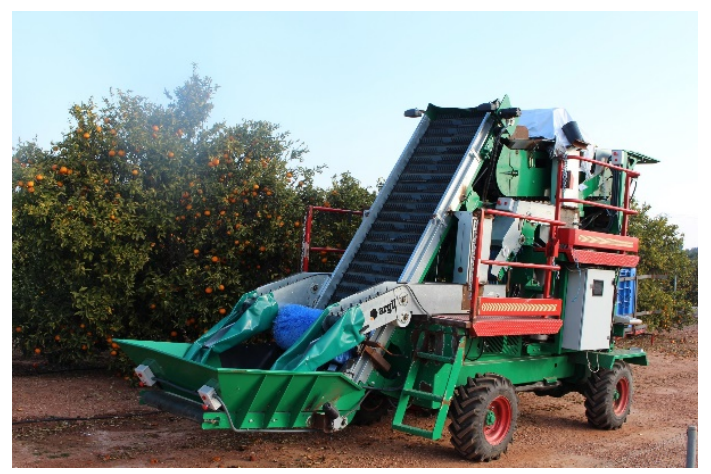

Figura 1. Prototipo de plataforma móvil.

La recolección se llevó a cabo en una de las parcelas de cultivo de cítricos en las instalaciones del IVIA. Para el ensayo se recolectaron un conjunto de árboles pertenecientes a tres calles contiguas. No se recolectaron filas de cultivo completas ya que la configuración de plantación no permitía el giro del prototipo al final de cada calle. En la figura 2 se muestra el esquema de recolección en la parcela.

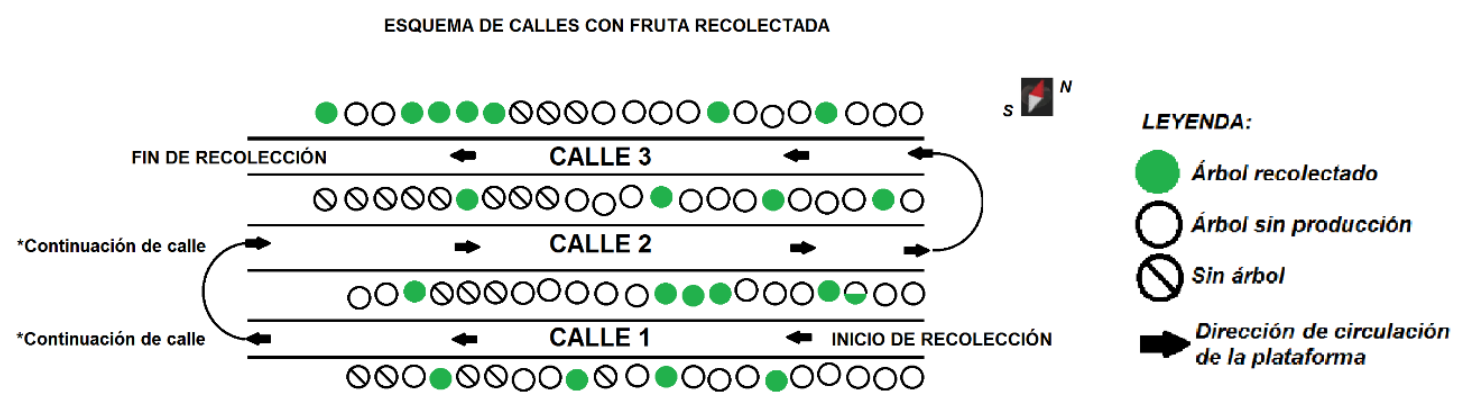

Figura 2. Esquema de recolección en parcela de IVIA. 


\section{CONGRESO IBÉRICO DE AGROINGENIERÍA \\ X CONGRESSO IBÉRICO DE AGROENGENHARIA \\ 3 - 6 septiembre 2019, Huesca - España}

En la plataforma móvil, la información se adquiere individualmente para cada fruta utilizando un sistema de visión instalado en la parte superior del prototipo. Las propiedades externas, como el área, el diámetro y el índice de color de la fruta se obtienen de las imágenes captadas en tiempo real durante la recolección. Los datos de ubicación geográfica se adquieren a través de un sistema global de navegación por satélite (GNSS) instalado en el prototipo. La información es almacenada en diferentes archivos en el ordenador industrial del prototipo.

Previo al comienzo de la recolección, el sistema de visión requiere insertar manualmente el valor numérico del diámetro según el cual se va a llevar a cabo la clasificación en campo en 2 categorías. En este ensayo, el valor insertado fue de $64 \mathrm{~mm}$. Se consideró que aquella fruta cuyo diámetro fuera superior a $64 \mathrm{~mm}$ alcanzaba una alta calidad con destino el mercado en fresco, enviándose al contenedor exterior dispuesto sobre la plataforma ('Output 1'), y el resto, catalogadas de calidad inferior y, por tanto, con destino a industria, fueron enviadas al contenedor interior ('Output 2').

\section{Resultados y discusión}

La base de datos espacial se creó vinculando la información de las propiedades externas de los cítricos adquiridas en el momento de inspección (no en el momento de recolección del árbol) con los datos de ubicación del GPS (figura 3).

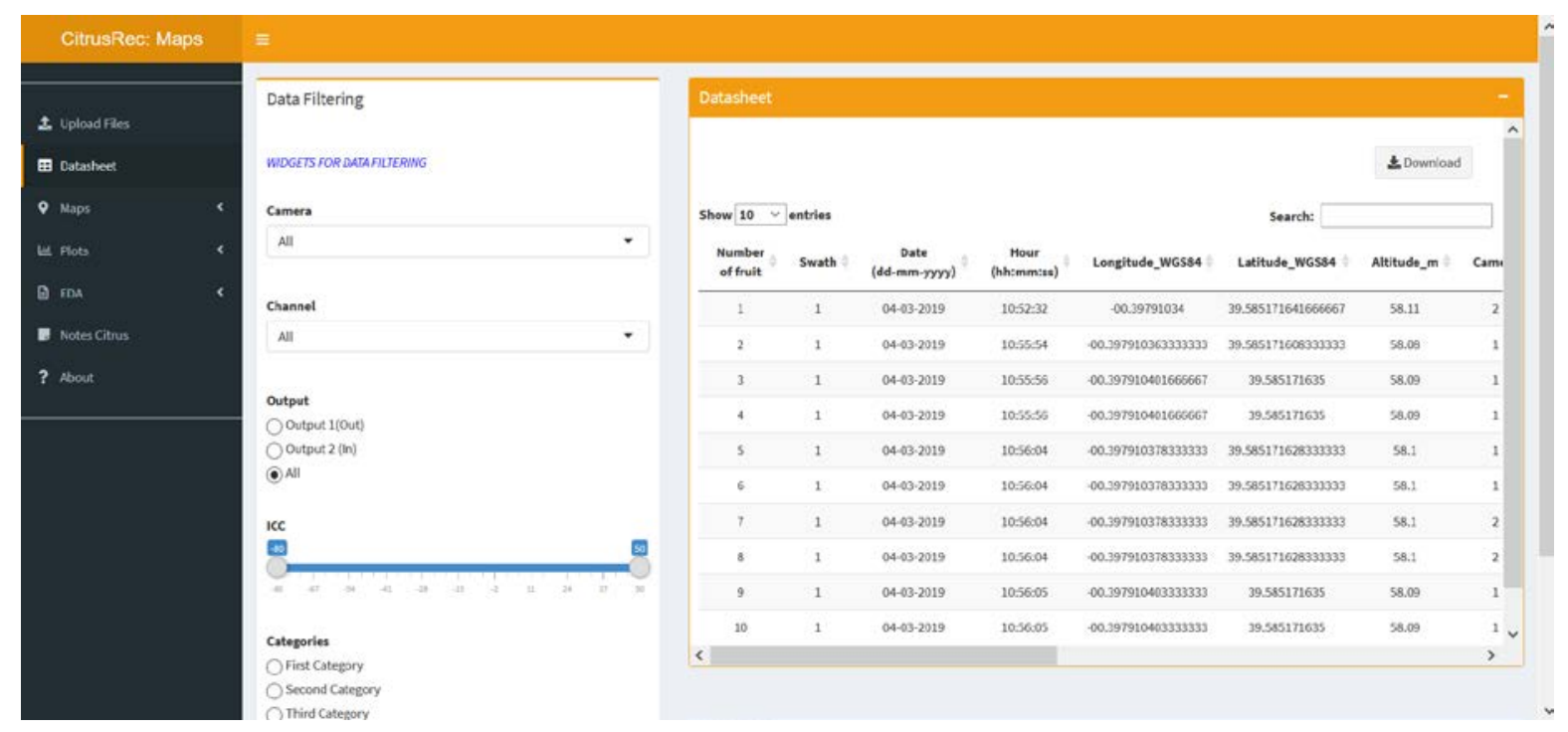

Figura 3. Base de datos espacial.

Para esta prueba las variables informativas fueron: número de cámara que adquiere las imágenes (denominado 'Camera'), carril que lleva la fruta hasta el sistema de inspección (denominado 'Channel') y contenedor al que se envía la fruta después de la inspección (denominado 'Output'). Respecto a las variables de calidad se tienen: diámetro, área, peso e índice de color.

Las opciones disponibles para filtrar los datos según las variables medidas permiten para este caso, por ejemplo, separar la información de las frutas cuyo destino es el consumo en fresco o la industria.

Cada mapa visualiza la fruta recolectada de acuerdo con cada una de las variables seleccionadas a nivel de calle y parcela para estos datos. Por tanto, con la información de este ensayo en la aplicación se crean tres mapas: uno denominado 'mapa de producción' donde se muestra la producción en función de la variable 'output', uno denominado 'mapa de color' 


\section{CONGRESO IBÉRICO DE AGROINGENIERÍA \\ X CONGRESSO IBÉRICO DE AGROENGENHARIA \\ 3 - 6 septiembre 2019, Huesca - España}

donde se muestra la producción en función de la variable 'índice de color' y uno denominado 'mapa de calibre' donde se muestra la producción según la variable 'diámetro'. En la figura 4 se muestra un ejemplo de un mapa que representa la información georreferenciada de las frutas recolectadas.

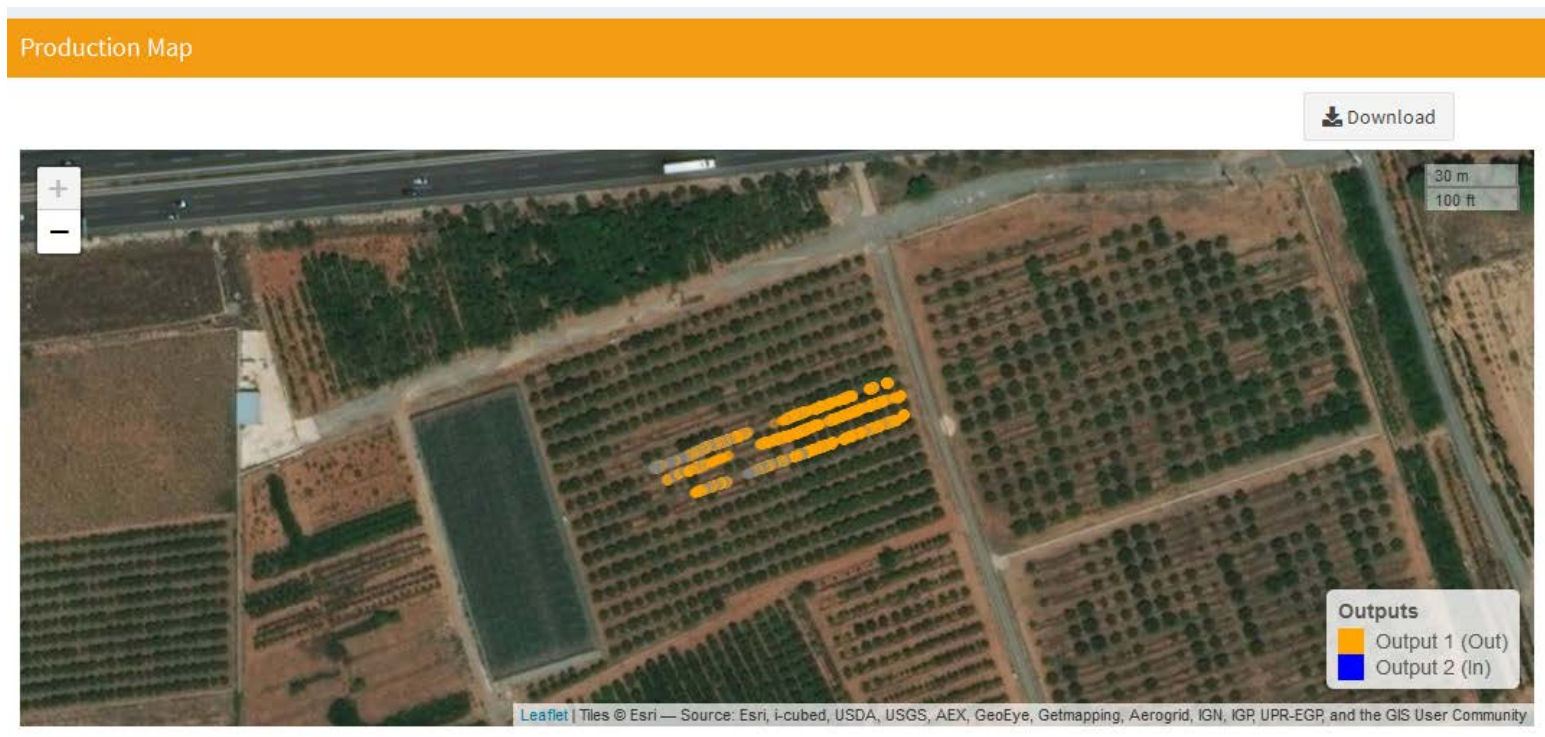

Figura 4. Mapa creado en la aplicación: mapa de producción.

Como se aprecia en la figura 4, dentro de cada fila se representa la ubicación concreta de cada dato. Con ello, se muestran áreas donde ninguna fruta ha pasado por el sistema de visión, lo cual índica la existencia de zonas con rendimiento nulo. En las tablas complementarias a cada uno de los mapas se muestra la información de producción en kilogramos, en unidades de fruta y en tanto por ciento del total de producción en función de la variable correspondiente para cada calle y para el total de calles donde se ha recolectado fruta. A modo de ejemplo, en la tabla 1 se muestra las tablas adyacentes al mapa de producción.

\begin{tabular}{|c|c|c|c|c|c|c|c|c|}
\hline Swath $\triangleq$ & Production $(\mathrm{Kg}) /$ Swath $\nRightarrow$ & \multicolumn{2}{|c|}{ Production(\%)/Swath } & \multicolumn{2}{|c|}{$\begin{array}{c}\text { Production } \\
\text { Output1 }(\mathrm{Kg}) / \mathrm{Swath}\end{array}$} & $\begin{array}{c}\text { Production } \\
\text { Output1(\%)/Swath }\end{array}$ & $\begin{array}{c}\text { Production } \\
\text { Output2(Kg)/Swath }\end{array}$ & $\begin{array}{c}\text { Production } \\
\text { Output } 2(\%) / \text { Swath }\end{array}$ \\
\hline 1 & 501.1 & \multicolumn{2}{|c|}{29.83} & \multicolumn{2}{|c|}{440.56} & 26.23 & 60.54 & 3.6 \\
\hline 2 & 558.87 & \multicolumn{2}{|c|}{33.27} & \multicolumn{2}{|c|}{519.42} & 30.92 & \multicolumn{2}{|r|}{2.35} \\
\hline 3 & 619.76 & \multicolumn{2}{|c|}{36.9} & \multicolumn{2}{|c|}{491.44} & 29.26 & \multicolumn{2}{|l|}{128.32} \\
\hline \multicolumn{9}{|c|}{ Showing 1 to 4 of 4 entries } \\
\hline Swath $\Rightarrow$ & $\begin{array}{c}\begin{array}{c}\text { Number of } \\
\text { fruits(Uts)/Swath }\end{array} \\
\text { frui }\end{array}$ & $\begin{array}{l}\text { Number of } \\
\text { uits(\%)/Swath }\end{array}$ & \multicolumn{2}{|c|}{$\begin{array}{l}\text { Number of fruits } \\
\text { Output1(Uts)/Swath }\end{array}$} & \multicolumn{2}{|c|}{$\begin{array}{c}\text { Number of fruits } \\
\text { Output1(\%)/Swath }\end{array}$} & $\begin{array}{l}\text { nber of fruits } \\
\text { ut2(Uts)/Swath }\end{array}$ & $\begin{array}{c}\text { Number of fruits } \\
\text { Output2(\%)/Swath }\end{array}$ \\
\hline 3 & 4153 & 40.21 & & 66 & & 26.78 & 1387 & 13.43 \\
\hline TOTAL & 10327 & 100 & & 29 & & 76.78 & 2398 & 23.22 \\
\hline
\end{tabular}

Showing 1 to 4 ot 4 entries

Figura 5. Tablas complementarias al mapa de producción creadas por la aplicación. 


\section{CONGRESO IBÉRICO DE AGROINGENIERÍA \\ X CONGRESSO IBÉRICO DE AGROENGENHARIA \\ 3 - 6 septiembre 2019, Huesca - España}

Comparando la información mostrada en el mapa de producción (figura 4) con el esquema de recolección (figura 2), se aprecia un desplazamiento longitudinal de los datos pues el prototipo inspecciona la fruta que ha sido recolectada de los árboles que se encuentran en la zona de trabajo posicionada en la parte delantera del prototipo.

La aplicación muestra de manera clara aquellas zonas con nula productividad, esto es, donde ninguna fruta ha pasado por el sistema de visión y los puntos de principio y fin de cada calle por donde se lleva a cabo la recolección.

En función del proceso de clasificación llevado a cabo en la plataforma móvil, en el zoom sobre el mapa de producción mostrado en la figura 5 se aprecian amplias zonas donde los frutos se han considerado válidos (áreas compuestas mayoritariamente con datos de color anaranjado) y algunas zonas de menor tamaño donde los frutos se han considerado no válidos (áreas compuestas mayoritariamente con datos de color azulado). De esta forma se representa la variabilidad espacial de los datos en función de la variable 'output'.

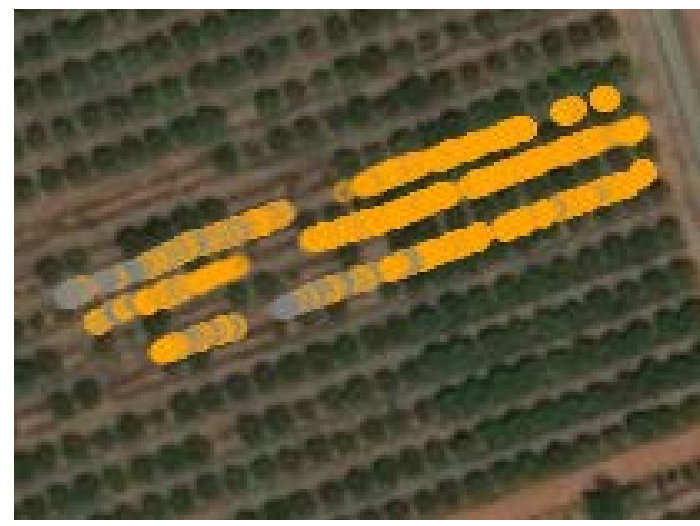

Figura 6. Zoom sobre el mapa de producción.

En la figura 6 se muestran los gráficos interactivos asociados al mapa de producción. En la gráfica de la izquierda se refleja la producción en función de la calle de cultivo y de la parcela. En la gráfica de la derecha se visualiza la producción en función de la variable 'output' por calle de cultivo y por parcela. Al interaccionar con los gráficos las etiquetas muestran la información cuantitativa relativa a cada variable.

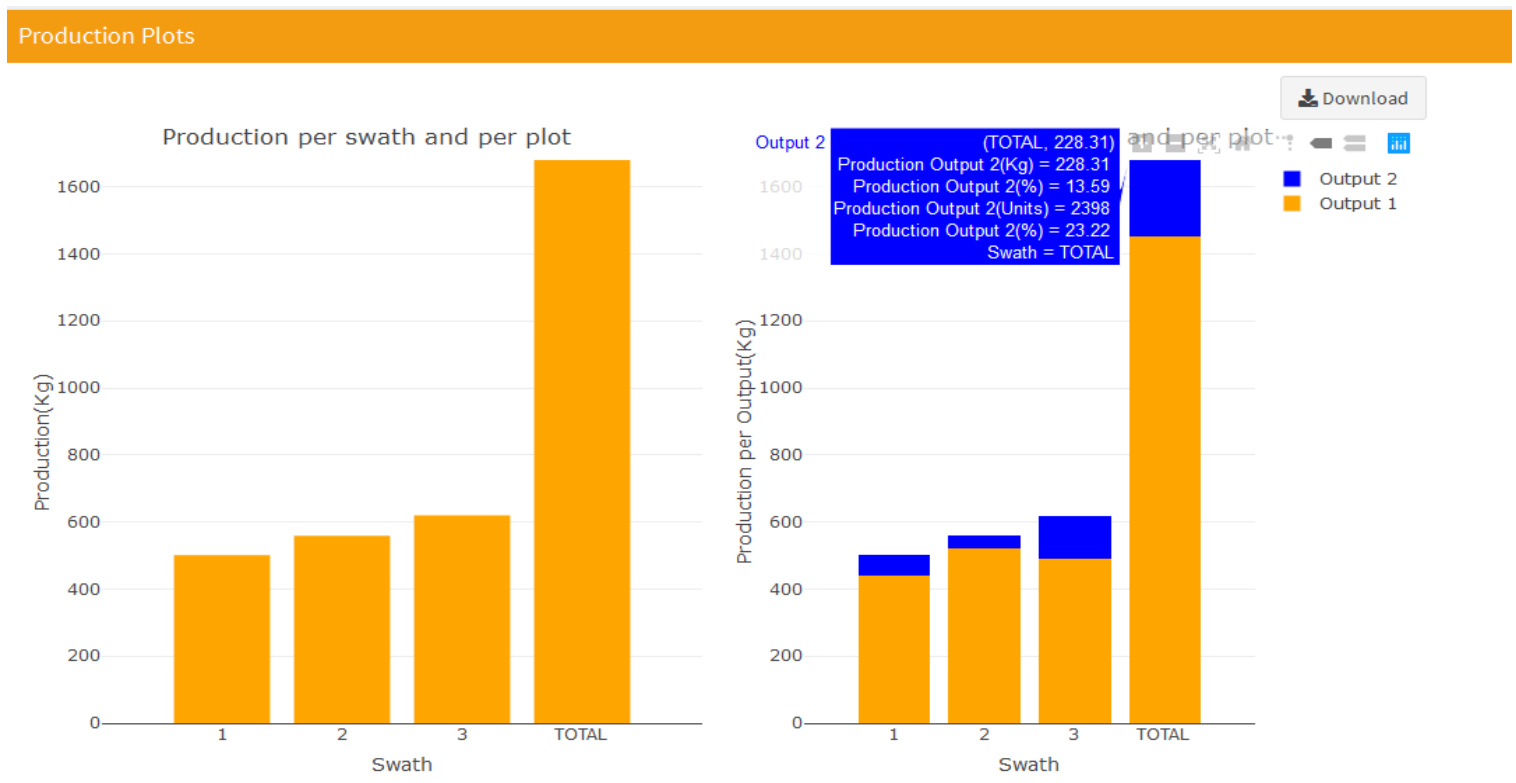

Figura 7. Gráfico interactivo asociado al mapa de producción. 


\section{CONGRESO IBÉRICO DE AGROINGENIERÍA \\ X CONGRESSO IBÉRICO DE AGROENGENHARIA \\ 3 - 6 septiembre 2019, Huesca - España}

\section{Conclusiones}

Se ha desarrollado una herramienta para generar mapas de rendimiento con información recopilada del cultivo que proporciona conocimiento práctico sobre la explotación agrícola. Esta aplicación presenta una importante ventaja: la creación de una base de datos integrando la información espacial con la información proporcionada por cualquier sensor o conjunto de sensores utilizando la variable tiempo como método de vinculación. La información contenida en esta base de datos es georreferenciada sobre un mapa en función de las variables medidas que se seleccionen y es analizada estadísticamente mostrando además la información cuantitativa en gráficos y tablas. La generación automática de informes permite hacer uso de esta información fuera de la aplicación.

Esta herramienta informática no almacena la información gestionada por lo que no infringe ninguna regulación, norma o ley relacionada con los derechos de la información y de su propiedad.

Esta aplicación web es capaz de simplificar al usuario el procesado de los datos y su análisis, obteniendo directamente información gráfica y cuantitativa expresada de manera comprensible a través de una aplicación web con interfaz intuitiva y visual. La herramienta proporciona información de trazabilidad en el campo pudiendo asociar cada dato con su ubicación.

Como proyección futura, se pretende ampliar este sistema para monitorizar y generar mapas de cualquier parámetro de cultivo como puede ser aplicación de fitosanitarios, detección de malezas, estado vegetativo, aplicación de nutrientes, aplicación de riego, entre otros. La combinación de la información referente al rendimiento de la recolección con otro tipo de información sobre el suelo y sobre el cultivo permitirá gestionar el campo de forma más eficiente.

\section{Agradecimientos}

Este trabajo se ha llevado a cabo dentro del proyecto "Aplicación de nuevas tecnologías para una estrategia integral de cosecha mecanizada de cítricos (CITRUSREC)" financiado por el Instituto Nacional de Investigaciones Agrícolas (INIA) y el Ministerio de Economía, Industria y Competitividad de España (proyectos RTA2014-00025-C05-01 y RTA2014-00025-C05-05) y cofinanciado por el Fondo Europeo de Desarrollo Regional (FEDER). Ma Gyomar González tiene concedida una beca predoctoral FPI-INIA. Este trabajo ha sido posible gracias a la ayuda del personal del Centro de Agroingeniería del IVIA y del personal del IDAL.

\section{Referencias}

1. Ping, J. L., Dobermann, A. Processing of yield map data. Precision Agriculture. 2005, 6(2), (193-212).

2. Sudduth, K. A., Drummond, S. T. Yield editor. Agronomy Journal. 2007, 99(6), (1471-1482).

3. Dobermann, A., Ping, J. L., Adamchuk, V. I., Simbahan, G. C., Ferguson, R. B. Classification of crop yield variability in irrigated production fields. Agronomy Journal. 2003, 95(5), (1105-1120).

4. Mantovani, E. C., \& Magdalena, C. Manual de agricultura de precisión. Embrapa Milho e Sorgo-Livro científico (ALICE). 2014.

5. Zagórda, M., Walczykova, M. The application of various software programs for mapping yields in precision agriculture. In BIO Web of Conferences. EDP Sciences. 2018, 10, (01018).

6. Uva, M., Campanella, O. AP-SIG: un SIG con funciones específicas para Agricultura de Precisión. In XI Workshop de Investigadores en Ciencias de la Computación. 2009.

7. Tan, L., Haley, R., Wortman, R. Cloud-based harvest management system for specialty crops. In IEEE Fourth Symposium on Network Cloud Computing and Applications (NCCA). 2015, (91-98).

8. Ampatzidis, Y., Tan, L., Haley, R., Whiting, M. D. Cloud-based harvest management information system for hand-harvested specialty crops. Computers and Electronics in Agriculture. 2016, 122, (161-167). 


\section{CONGRESO IBÉRICO DE AGROINGENIERÍA \\ X CONGRESSO IBÉRICO DE AGROENGENHARIA \\ 3 - 6 septiembre 2019, Huesca - España}

9. Gonzalez-de-Santos, P., Ribeiro, A., Fernandez-Quintanilla, C., Lopez-Granados, F., Brandstoetter, M., Tomic, S., Perez-Ruiz, M. Fleets of robots for environmentally-safe pest control in agriculture. Precision Agriculture. 2017, 18(4), (574-614).

10. Leroux, C., Jones, H., Pichon, L., Guillaume, S., Lamour, J., Taylor, J., Tisseyre, B. GeoFIS: una herramienta de código abierto para la toma de decisiones para datos de agricultura de precisión. Agricultura. 2018, 8 (6), (73).

11. Rovira-Más, F., Sáiz-Rubio, V. Crop biometric maps: The key to prediction. Sensors. 2013, 13(9), (12698-12743).

12. Tan, L., Wortman, R. Cloud-based monitoring and analysis of yield efficiency in precision farming. In Proceedings of the 2014 IEEE 15th International Conference on Information Reuse and Integration. IEEE. 2014, (163-170). 